\title{
What can Parkinson's disease teach us about COVID-19?
}

\author{
Philip W. Tipton, Zbigniew K. Wszolek \\ Department of Neurology, Mayo Clinic, Jacksonville, Florida, United States
}

The pandemic potential of coronaviruses (CoV) has now been realised with coronavirus disease 19 (COVID-19) caused by SARS-CoV-2 (Severe Acute Respiratory Syndrome Coronavirus 2). This infection began in December 2019 in Wuhan, China before spreading across the globe in a matter of weeks. At the time of this submission, there were more than 1.8 million cases and 119,000 deaths worldwide [1]. The global health community is working furiously to gain a better understanding of COVID-19 disease mechanisms and to develop effective preventative and treatment strategies. Elderly individuals and those with comorbidities, especially cardiovascular, are at an increased risk of infection and poor outcomes. Patients with Parkinson's disease (PD) often fall into this high-risk category, but may be at even greater risk due to SARS-CoV-2 neuronal tropism. We here highlight evidence to support this claim, and propose the study of two widely used PD medications for the treatment of COVID-19.

Much of our knowledge regarding SARS-CoV-2 comes from previous studies of other human coronaviruses (SARS- $\mathrm{CoV}$ and MERS-CoV), which have caused much smaller epidemics.

Information is now emerging about SARS-CoV-2 characteristics including the risk of acute respiratory distress syndrome (ARDS). Current evidence suggests that ARDS results from direct lung invasion through the angiotensin-converting enzyme 2 (ACE2) receptor, which is expressed on lung alveolar cells [2]. A study of rodents identified widespread neuronal ACE2 expression in brainstem cardiorespiratory neurons, motor cortex, the raphe nucleus, and others [3]. While ACE2's role in the brain is incompletely understood, this diverse CNS expression pattern may provide SARS-CoV-2, and other CoVs, with a 'port of entry' into the CNS.

There are reports of neurological symptoms in patients with COVID-19. These are mostly presented as sequelae secondary to ARDS, e.g. hypoxic encephalopathy in $20 \%$ of patients [4]. However, there may be a direct relationship between ARDS and the nervous system. Neuroinvasion has been documented for many CoVs, including SARS-CoV, MERS-CoV, human CoV-OC43 (HCoV-OC43), HCoV-229E, mouse hepatitis virus (MHV), and porcine hemagglutinating encephalomyelitis coronavirus (HEV) [5]. Rodent models have shown that SARS-CoV and MERS-CoV can enter the brain after intranasal administration [5]. HEV67, which shares $91 \%$ homology with $\mathrm{HCoV}-\mathrm{OC} 43$, gains access to the CNS via trans-synaptic transfer from peripheral nerve terminals [5]. The avian influenza virus also spreads in this manner, and has been traced from the vagus nerve to the solitary nucleus and nucleus ambiguus in canines [6]. Involvement of these cardiorespiratory centres may result in a CNS-mediated component of COVID-19 cardiorespiratory manifestations.

The latest evidence indicates that COVID-19 symptoms are most severe in elderly individuals with various comorbidities such as hypertension, cardiovascular disease, diabetes mellitus, and renal disease [4]. This is especially concerning from a neurologist's perspective because age is the strongest risk factor for a variety of neurodegenerative diseases, including PD. Moreover, comorbid conditions accumulate with increasing age. It is unclear whether PD confers direct vulnerability to SARS-CoV-2, but parkinsonism is a well-known consequence of several viral encephalitides. In 1985, Fishman et al. reported that MHV-A59 has a selective affinity for the basal ganglia [7]. A subsequent study found enhanced antibody responses to different $\mathrm{CoV}$ forms in the cerebrospinal fluid of PD patients [8].

These findings warrant further investigation into SARS- CoV-2 vulnerability in PD.

Adamantane derivatives represent a class of medications that block NMDA receptor activity, and are effective at decreasing excess neuronal activity that may lead to excitotoxicity and unwanted neurological symptoms. Amantadine belongs to this class. It is an effective treatment for patients with PD who have levodopa-induced motor complications. Memantine is also an adamantane derivative most often used to slow the rate of cognitive decline in patients with dementia, including those with PD.

Some adamantane derivates are effective antiviral agents. Amantadine was initially marketed as a treatment against influenza A. By blocking the matrix-2 (M2) protein ion channel, amantadine inhibits viral uncoating within host cell endosomes [9]. Bananin is an adamantane derivative that acts against the SARS coronavirus by blocking the enzyme helicase, which is

Corresponding Author: Philip W. Tipton, Department of Neurology, Mayo Clinic, 4500 San Pablo Rd., Jacksonville, FL 32224, USA,

e-mail: tipton.philip@mayo.edu 
critical for viral nucleic acid replication [9]. Memantine may inhibit ion channel activity of the $\mathrm{HCoV}-\mathrm{OC} 43$ protein E, similar to amantadine's action on M2 protein, or inhibit viral helicase activity like bananin [9]. It is unknown whether amantadine or memantine are effective antiviral treatments of SARS-CoV-2.

There is a growing amount of data supporting the treatment of COVID-19 with repurposed non-antiviral agents, such as chloroquine, hydroxychloroquine, and azithromycin $[10,11]$. It is vital to consider possible serious side effects (e.g. QT interval prolongation) when administering these medications, especially in older patients with cardiac comorbidities [12].

We should consider repurposing other medications, especially those with known antiviral properties such as amantadine and memantine. These medications are inexpensive, widely used, and have well-known side effect profiles that are relatively mild compared to other potential COVID-19 treatments such as hydroxychloroquine. Their ability to cross the blood/brain barrier also provides direct CNS access for potential neuroprotection. In this manner, amantadine and memantine may have a role to play in the prevention and/or acute treatment of COVID-19.

Elderly individuals are at increased risk for a more severe disease course and worse outcomes [4]. Levodopa-induced motor complications and dementia tend to be later manifestations of PD. Consequently, amantadine and memantine are typically prescribed to older, more at-risk, patients. Therefore, these individuals are ideal candidates for retrospective studies to determine whether amantadine and/or memantine can reduce one's risk of infection with COVID-19 or diminish symptom severity. Patients receiving amantadine and/or memantine may experience QT interval prolongation. We recommend careful ECG assessment at treatment onset and serial monitoring for individuals receiving one or more of these medications, especially if combined with chloroquine.

The COVID-19 pandemic has overwhelmed the healthcare systems of many countries. At this time, our most effective weapon to prevent healthcare system overload in further countries is a combination of social distancing and thorough hand hygiene. Sporting events have been cancelled, schools have converted to computer-based formats, and countless individuals must stay at home rather than go to work, sometimes even without pay. Each day of increasing viral spread brings a greater burden to our economy and way of life.

We must explore all feasible options for preventing and treating COVID-19. Trial repurposing of medications that are inexpensive, and readily available, is a low risk and cost-effective approach. We propose amantadine and memantine as two potential candidates.

\section{Financial disclosure/conflict of interest}

Dr. Tipton reports no disclosures or conflicts of interest. Dr. Wszolek receives support from the Mayo Clinic Center for Regenerative Medicine, gifts from The Sol Goldman Charitable
Trust, and the Donald G. and Jodi P. Heeringa Family, the Haworth Family Professorship in Neurodegenerative Diseases fund, and The Albertson Parkinson's Research Foundation. He serves as PI or Co-PI on Biogen, Inc. (228PD201), and Biohaven Pharmaceuticals, Inc. (BHV4157-206 and BHV3241-301) grants. He serves as PI of the Mayo Clinic American Parkinson's Disease Association (APDA) Information and Referral Center. He is a co-editor-in-chief of Neurologia i Neurochirurgia Polska (the Polish Journal of Neurology and Neurosurgery).

Author roles: PA Tipton - manuscript composition and formatting; ZK Wszolek - manuscript editing.

\section{Acknowledgements}

Mayo Clinic is an American Parkinson's Disease Association (APDA) Information and Referral Center and APDA Center for Advanced Research.

\section{Glossary}

ACE2 - Angiotensin-converting enzyme 2

ARDS - Acute respiratory distress syndrome

$\mathrm{CoV}$ - Coronavirus

COVID-19 - coronavirus 19

ECG - electrocardiography

HEV - hemagglutinating encephalomyelitis coronavirus

M2 - Matrix-2

MHV - mouse hepatitis virus

PD - Parkinson's disease

SARS-CoV-2 - Severe Acute Respiratory Syndrome Coronavirus 2

Funding sources for the study: None

\section{References}

1. World Health Organization. Coronavirus disease (COVID-19) Pandemic. https://www.who.int/emergencies/diseases/novel-coronavirus-2019. (April 14, 2020).

2. Zhou $P$, Yang $X L$, Wang $X G$, et al. A pneumonia outbreak associated with a new coronavirus of probable bat origin. Nature. 2020; 579(7798): 270-273, doi: 10.1038/s41586-020-2012-7, indexed in Pubmed: 32015507.

3. Doobay MF, Talman LS, Obr TD, et al. Differential expression of neuronal ACE2 in transgenic mice with overexpression of the brain renin-angiotensin system. Am J Physiol Regul Integr Comp Physiol. 2007; 292(1): R373-R381, doi: 10.1152/ajpregu.00292.2006, indexed in Pubmed: 16946085.

4. Chen T, Wu Di, Chen H, et al. Clinical characteristics of 113 deceased patients with coronavirus disease 2019: retrospective study. BMJ. 2020; 368: m1091, doi: 10.1136/bmj.m1091, indexed in Pubmed: 32217556.

5. Li YC, Bai WZ, Hashikawa T, et al. The neuroinvasive potential of SARS-CoV2 may play a role in the respiratory failure of COVID-19 patients. 
J Med Virol. 2020 [Epub ahead of print], doi: 10.1002/jmv.25728, indexed in Pubmed: 32104915.

6. Matsuda K, Park $\mathrm{CH}$, Sunden $\mathrm{Y}$, et al. The vagus nerve is one route of transneural invasion for intranasally inoculated influenza a virus in mice. Vet Pathol. 2004; 41(2): 101-107, doi: 10.1354/vp.41-2101, indexed in Pubmed: 15017022.

7. Fishman PS, Gass JS, Swoveland PT, et al. Infection of the basal ganglia by a murine coronavirus. Science. 1985; 229(4716): 877-879, doi: 10.1126/science.2992088, indexed in Pubmed: 2992088.

8. Fazzini E, Fleming J, Fahn S. Cerebrospinal fluid antibodies to coronavirus in patients with Parkinson's disease. Mov Disord. 1992; 7(2): 153-158, doi: 10.1002/mds.870070210, indexed in Pubmed: 1316552.

9. Brison $\mathrm{E}$, Jacomy $\mathrm{H}$, Desforges $\mathrm{M}$, et al. Novel treatment with neuroprotective and antiviral properties against a neuroinvasive human respiratory virus. J Virol. 2014; 88(3): 1548-1563, doi: 10.1128/ JVI.02972-13, indexed in Pubmed: 24227863.

10. Gautret $P$, Lagier JC, Parola P, et al. Hydroxychloroquine and azithromycin as a treatment of COVID-19: results of an open-label non-randomized clinical trial. Int J Antimicrob Agents. 2020 [Epub ahead of print]: 105949, doi: 10.1016/j.ijantimicag.2020.105949, indexed in Pubmed: 32205204.

11. Gao J, Tian Z, Yang Xu. Breakthrough: Chloroquine phosphate has shown apparent efficacy in treatment of COVID-19 associated pneumonia in clinical studies. Biosci Trends. 2020; 14(1): 72-73, doi: 10.5582/bst.2020.01047, indexed in Pubmed: 32074550.

12. Cortegiani A, Ingoglia $\mathrm{G}$, Ippolito $\mathrm{M}$, et al. A systematic review on the efficacy and safety of chloroquine for the treatment of $\mathrm{CO}$ VID-19. J Crit Care. 2020 [Epub ahead of print], doi: 10.1016/i. jcrc.2020.03.005, indexed in Pubmed: 32173110. 